\title{
Comparison of methods of alert acknowledgement by critical care clinicians in the ICU setting
} \author{
Ognjen Gajic ${ }^{5}$, Brian W Pickering ${ }^{2}$, Vitaly Herasevich ${ }^{\text {Corresp. } 2}$ \\ ${ }^{1}$ Medical Scientist Training Program, Mayo Clinic, Rochester, Minnesota, United States of America \\ 2 Department of Anesthesiology, Mayo Clinic, Rochester, Minnesota, United States of America \\ 3 Department of Internal Medicine, Mayo Clinic, Rochester, Minnesota, United States of America \\ 4 Mayo Medical School, Mayo Clinic, Rochester, Minnesota, United States of America \\ 5 Division of Pulmonology and Critical Care Medicine, Mayo Clinic, Rochester, Minnesota, United States of America \\ Corresponding Author: Vitaly Herasevich \\ Email address: Herasevich.Vitaly@mayo.edu
}

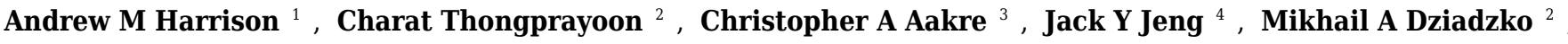

Background. Electronic Health Record (EHR)-based sepsis alert systems have failed to demonstrate improvements in clinically meaningful endpoints. However, the effect of implementation barriers on the success of new sepsis alert systems is rarely explored.

Objective. To test the hypothesis time to severe sepsis alert acknowledgement by critical care clinicians in the ICU setting would be reduced using an EHR-based alert acknowledgement system compared to a text paging-based system. Study Design. In one arm of this simulation study, real alerts for patients in the medical ICU were delivered to critical care clinicians through the EHR. In the other arm, simulated alerts were delivered through text paging. The primary outcome was time to alert acknowledgement. The secondary outcomes were a structured, mixed quantitative/qualitative survey and informal group interview. Results. The alert acknowledgement rate from the severe sepsis alert system was $3 \%(\mathrm{~N}=148)$ and $51 \%(\mathrm{~N}=156)$ from simulated severe sepsis alerts through traditional text paging. Time to alert acknowledgement from the severe sepsis alert system was median 274 minutes $(\mathrm{N}=5)$ and median 2 minutes $(\mathrm{N}=80)$ from text paging. The response rate from the EHR-based alert system was insufficient to compare primary measures. However, secondary measures revealed important barriers.

Conclusion. Alert fatigue, interruption, human error, and information overload are barriers to alert and simulation studies in the ICU setting. 
1 Comparison of methods of alert acknowledgement by critical care clinicians in the ICU

2 setting

3 Andrew M. Harrison, PhD; ${ }^{1}$ Charat Thongprayoon, MD;² Christopher A. Aakre, MD; ${ }^{3}$ Jack Y.

4 Jeng, MD, MBA; ${ }^{4}$ Mikhail A. Dziadzko, MD, PhD;² Ognjen Gajic, MD, MSc; ${ }^{5}$ Brian W. Pickering,

$5 \mathrm{MB}, \mathrm{BCh}, \mathrm{MSc}^{2}$ Vitaly Herasevich, MD, $\mathrm{PhD}^{2}$

6 Affiliations

7 1. Medical Scientist Training Program, Mayo Clinic, Rochester, MN

8 2. Department of Anesthesiology, Mayo Clinic, Rochester, MN

9 3. Department of Internal Medicine, Mayo Clinic, Rochester, MN

10 4. Mayo Medical School, Mayo Clinic, Rochester, MN

11 5. Division of Pulmonology and Critical Care Medicine, Mayo Clinic, Rochester, MN

12 Institution: This work was performed at Mayo Clinic in Rochester, MN, USA

13 Correspondence

14 Vitaly Herasevich, MD, PhD

15 Mayo Clinic, Department of Anesthesiology

16200 First Street SW, Rochester, MN 55905

17 E-Mail: Herasevich.Vitaly@mayo.edu

18 Phone: +1 507-255-4055; Fax: +1 507-255-4267 
19 ABSTRACT

20 Background. Electronic Health Record (EHR)-based sepsis alert systems have failed to 21 demonstrate improvements in clinically meaningful endpoints. However, the effect of 22 implementation barriers on the success of new sepsis alert systems is rarely explored.

23 Objective. To test the hypothesis time to severe sepsis alert acknowledgement by critical care 24 clinicians in the ICU setting would be reduced using an EHR-based alert acknowledgement 25 system compared to a text paging-based system.

26 Study Design. In one arm of this simulation study, real alerts for patients in the medical ICU 27 were delivered to critical care clinicians through the EHR. In the other arm, simulated alerts 28 were delivered through text paging. The primary outcome was time to alert acknowledgement. 29 The secondary outcomes were a structured, mixed quantitative/qualitative survey and informal 30 group interview.

31 Results. The alert acknowledgement rate from the severe sepsis alert system was $3 \%(N=148)$ 32 and $51 \%(\mathrm{~N}=156)$ from simulated severe sepsis alerts through traditional text paging. Time to 33 alert acknowledgement from the severe sepsis alert system was median 274 minutes $(\mathrm{N}=5)$ and 34 median 2 minutes $(\mathrm{N}=80)$ from text paging. The response rate from the EHR-based alert system 35 was insufficient to compare primary measures. However, secondary measures revealed 36 important barriers.

37 Conclusion. Alert fatigue, interruption, human error, and information overload are barriers to 38 alert and simulation studies in the ICU setting.

39 Word count: 215 


\section{INTRODUCTION}

41 Electronic health record (EHR)-based, automated sepsis alert systems have failed to 42 demonstrate improvements in clinically meaningful endpoints, such as Intensive Care Unit 43 (ICU)/hospital length of stay (LOS) and mortality (Hooper et al. 2012; LaRosa et al. 2012; 44 Nelson et al. 2011; Sawyer et al. 2011). This includes studies of ICU-specific and non-ICU 45 specific alert systems. This also includes studies ranging in variation regarding degree of 46 distinction between the detection of sepsis, severe sepsis, and/or septic shock (Dellinger et al. 47 2013). Clinically meaningful endpoints range from compliance with the international Surviving 48 Sepsis Campaign (SSC) guidelines to hospital LOS, ICU LOS, and mortality. There are ICU49 based and hospital wide means to trigger an alert for the early recognition of sepsis. Most EHRs 50 now have a built in system to support this alert

52 Time to alert acknowledgement has been validated as one proxy for time to recognition of sepsis by critical care clinicians (Dziadzko et al. 2016). The failure of EHR-based, automated sepsis alert systems to be directly correlated with improvements in clinically meaningful endpoints is frequently attributed to limitations of detection algorithms and/or the need for clinical decision support (CDS) systems (Semler et al. 2015). Human factors, such as the impact of workflow changes or the influence of method of alert delivery, are known to be barriers to the implementation of new alert systems in the clinical setting (Harrison et al. 2015a).

As "alarm hazards" have been ranked as the top health technology hazard in the United States (ECRI-Institute 2013), it is important to explore the effect of implementation of new alert systems 62 on workflow changes and other human factors in the clinical setting. Despite outcome 63 improvements in recent decades (Kaukonen et al. 2014), sepsis remains one of the most 64 expensive in-hospital conditions (Torio CM 2013). As one of the most technologically sophisticated hospital environments, the critical care setting serves as a model to explore the 
66 impact of implementation of new alert systems. Despite these technological advances, including

67 widespread utilization of text messaging and smartphones by clinicians, text paging remains

68 standard practice in the hospital setting (HIMSS Analytics 2016). We hypothesized that time to

69 severe sepsis alert acknowledgement by critical care clinicians in the ICU setting would be

70 reduced using an EHR-based alert acknowledgement system compared to a text paging-based

71 system.

72

73 METHODS

$74 \quad$ Study design and setting

75 This study was performed in February 2015 in the medical ICU at Mayo Clinic in Rochester, 76 MN, USA (Figure 1). This study was not performed in a laboratory setting or simulation 77 environment. This medical ICU has been described previously as a benchmark for evaluation of institutional performance (Afessa et al. 2005). Severe sepsis alerts were delivered to critical care clinicians, including attending physicians, fellows, residents, and nurse practitioners/physician assistants (NPs/PAs) using traditional HIPAA-compliant text paging. This study was approved by the Mayo Clinic Institutional Review Board (IRB) for clinician-participant enrollment by oral consent.

\section{Study participants and medical ICU workflow}

The medical ICU at Mayo Clinic consists of 2 physically adjacent 12-bed units, in close proximity to a nearby 9-bed respiratory care unit (RCU). For any given month, there are approximately 15 critical care attending physicians, 6 critical care fellows, 4 postgraduate year 3 internal medicine residents (PGY-3), 6 PGY-1 interns, and 9 dedicated medical ICU NPs/PAs. There are 2 shifts: 6am to $6 \mathrm{pm}(\mathrm{AM})$ and $6 \mathrm{pm}$ to $6 \mathrm{am}(\mathrm{PM})$. On any given day, the morning shift is further divided into 2 teams. Team 1 is assigned to the majority of the medical ICU patients. Team 2 is assigned the remaining patients, as well as the RCU, which is further staffed by an additional 
92 fellow and dedicated NP/PA from the same group of approximately 40 clinicians in the medical

93 ICU that month.

94

95 AWARE (Ambient Warning and Response Evaluation)

96 AWARE is the ICU-specific EHR system used in this study for patient viewer/monitoring. It was

97 developed at Mayo Clinic and has been in routine clinical use in the medical ICU at Mayo Clinic

98 since July 2012 (Pickering et al. 2015; Pickering et al. 2010). AWARE has been demonstrated

99 to improve clinician task load, errors of cognition, and performance (Ahmed et al. 2011).

100 AWARE is accessible from every computer workstation in this medical ICU, including bedside

101 desktops, nursing stations, and clinician workrooms.

102

103

\section{Severe sepsis alert system}

104 The severe sepsis detection algorithm was developed at Mayo Clinic and implemented into 105 AWARE in December 2014 (Harrison et al. 2015b). Within AWARE, the severe sepsis alert system displays a passive, yellow alert icon when severe sepsis is detected. This yellow alert icon can also be activated manually by clinicians for specific patients, when severe sepsis is suspected, but not detected by the automated alert system. This yellow alert icon is automatically updated to display a passive, green alert icon within AWARE after completion of the 4 elements of the 3-hour Surviving Sepsis Campaign (SSC) bundle (Dellinger et al. 2013).

111 Once activated, the yellow alert icon will persist for at least 6 hours, unless completion of the 6 112 hour SSC bundle is detected (green alert icon) or manual deactivation by clinicians occurs. In 113 the context of prolonged severe sepsis and/or septic shock, the yellow alert icon can persist 114 indefinitely. The green alert icon automatically reverts back to "no sepsis detected" after 3 115 hours, unless additional automatic (or manual) activation occurs.

\section{Study procedures}


118 Clinicians agreed to participate in this study from February 02 through February 28 in 2015 . The

119 evening before each AM shift and the next PM shift, clinician participants for these upcoming 120 shifts received a detailed email reminder with instructions (Figure 2). The number of severe 121 sepsis system alerts per shift through AWARE (yellow or green icon alerts) was entirely 122 dependent on the number of septic patients in the medical ICU during any specific shift. 123 Clinician participants randomly received no more than 3 simulated severe sepsis alerts per shift 124 via text paging. In both cases, clinician participants were instructed to acknowledge all AWARE 125 and traditional text paging severe sepsis alerts by email response. The difference between the 126 time to severe sepsis alert activation in AWARE (or alert delivery via text page) and email 127 response was defined as the time to alert acknowledgement.

128

129

\section{Survey design}

130 To compare clinician satisfaction between the EHR-based alert acknowledgement system and 131 text paging-based system, clinician participants completed a structured, mixed quantitative/qualitative survey, upon completion of the severe sepsis alert acknowledgement portion of this study (full survey facsimile in Results, Figure 3). These questions were designed partially on existing clinician satisfaction surveys of alert methods for use in the hospital and critical care settings (Embi et al. 2008; Wagner et al. 1998).

\section{Statistical analysis}

138 Severe sepsis alert system data was extracted directly from AWARE using METRIC Data Mart, a near-real time relational database of the complete EHR, which was developed at Mayo Clinic and has been described previously (Herasevich et al. 2010). Data was queried using JMP Pro

141 (SAS Institute, Inc). Data collection and statistical analyses, such as the two-sided Student's t142 test and the Chi-squared test, were also performed in JMP Pro. For all statistical analyses, a $p$ - 
143 value of less than 0.05 was considered to be statistically significant. For all median values from

144 the survey results, interquartile range (IQR) was reported.

145

\section{RESULTS}

147 Prior to initiation of this study, a 1-day feasibility pilot was performed in January 2015 using 7 148 medical ICU clinicians. Based on the result of this feasibility study (data not shown), it was 149 determined that a sufficiently high clinician participant alert acknowledgement rate could be 150 obtained from both severe sepsis system alerts through AWARE (yellow or green icon alerts) 151 and simulated severe sepsis alerts through traditional text paging in the ICU setting. Based on 152 the results of this feasibility pilot, participant instructions were optimized (Figure 2).

153

154 Of the 40 clinicians staffing the medical ICU in February 2015, 13 (32\%) were recruited to 155 participate in this study. However, it was decided after 2 weeks (February 02 AM through February 15 PM) to prematurely terminate this study, due to sufficient statistical power for time to alert acknowledgement analysis, as well as feedback from clinician participants. As a result, it was necessary to exclude 1 NP/PA due to unavailability in the medical ICU during this shortened study period (RCU only). Ultimately, 12 clinicians participated: 5 NPs/PAs (out of 9), 3 attending physicians (out of 15), 2 fellows (out of 6), 2 PGY-3s (out of 4), and 0 PGY-1s (out of 6). The median number of potential AWARE alert acknowledgements per shift was 2 (IQR 1 to 4). The minimum and maximum numbers were 0 and 5 . The number of patients who trigged at least 1 severe sepsis system alert through AWARE (yellow or green icon alert) was 28 . Of the 28 shifts that occurred during this shortened study period, 23 shifts (82\%) were covered by at least 1 participant (Table 1).

167 The alert acknowledgement rate from the severe sepsis alert system through AWARE was $3 \%$ $168(\mathrm{~N}=148)$ and $51 \%(\mathrm{~N}=156)$ from simulated severe sepsis alerts through text paging (Table 2). 
169 Time to alert acknowledgement from the severe sepsis alert system through AWARE was 170 median 274 minutes $(\mathrm{N}=5)$ and median 2 minutes $(\mathrm{N}=80)$ from simulated severe sepsis alerts

171 through text paging. The 5 alert acknowledgements from the severe sepsis alert system through

172 AWARE came from only 3 clinician participants (NP/PA \#01, NP/PA \#04, and NP/PA \#05), while 173 all 12 participants acknowledged at least 1 simulated severe sepsis alert through text paging.

174

175 All participants completed a structured, mixed quantitative/qualitative survey. For the 176 quantitative portion of the survey (Figure 3), clinicians found alert by AWARE to be slightly less 177 disruptive than alert by text paging. Clinicians found acknowledgement of AWARE and text 178 paging alerts to be equally disruptive. When AWARE and text paging alerts were directly 179 compared, a clear preference for text paging for both "urgent" and "non-urgent" alerts was 180 present. When asked to "select one or more" (text paging, AWARE, email, phone call, text message, or other), the results for non-urgent alerts were mixed. However, when asked the same question for urgent alerts, the preference was once again clearly for text paging.

184 For the qualitative portion of the survey (Figure 4), 11 out of 12 clinician participants provided "at 185 least one suggestion for improving alert/notification delivery". Clinicians commented on inhomogeneous overall use of AWARE in the medical ICU, despite implementation several years prior (July 2012). Of the same 11 clinicians, 4 provided "any additional comments": the same 3 NPs/PAs who responded to at least 1 alert acknowledgement from the severe sepsis alert system through AWARE, as well as Attending \#03. A clear theme concerning alert fatigue, interruption, human error, and information overload was present.

192 An informal group interview in the form of a noon pizza party was held to thank all clinician 193 participants and gather additional feedback on the barriers to clinician participation and 194 engagement in this implementation study. The 4 clinicians who attended were once again the 
195 same 4 clinicians who provided "any additional comments" on the survey. The statements 196 regarding alert fatigue, interruption, human error, and information overload were reinforced, 197 despite a strong interest from these clinicians to participate. Regarding inhomogeneous overall 198 use of AWARE in the medical ICU, specific attention was drawn to a particular lack of interest 199 from residents to use AWARE, as well as a lack of interest from both residents and fellows to 200 participate in any research study during their required rotations through the medical ICU, 201 including implementation of the severe sepsis alert system.

202

\section{DISCUSSION}

204 We hypothesized that time to severe sepsis alert acknowledgement by critical care clinicians in 205 the ICU setting would be reduced using an EHR-based alert acknowledgement system 206 compared to a text paging-based system. Based on the limited alert acknowledgement 207 response rate using the severe sepsis alert system compared to traditional text paging, it was 208 not possible to answer this hypothesis. However, feedback from the structured, mixed quantitative/qualitative survey, as well as the informal group interview, provided invaluable insight into the sources of this limited acknowledgement response rate. Implementation barriers

211 included human factors, such as alert fatigue, interruption, human error, and information 212 overload.

213

214 With the implementation of increasingly sophisticated EHR systems, interest in the development 215 of novel automated detection and alert systems has increased (Bourgault et al. 2014). However, 216 investigation into best methods of alert delivery (text paging, EHR systems, email, phone calls, 217 and/or text messaging) for urgent and non-urgent alerts in the hospital setting is limited (Gill et 218 al. 2012). Investigation into the most appropriate clinician for alert delivery is also limited (Zhang 219 et al. 2003). Monitoring and alert systems have been developed for patient use in the home 220 setting (Steinman et al. 2011; Tchalla et al. 2012). However, there has been comparatively 
221 limited investigation into methods of alert delivery to clinicians in the hospital setting (Loo et al. 222 2011). Interestingly, many of these studies have been performed in the geriatric patient 223 population, but not in the ICU setting, where the average patient age is often 65 or older 224 (Seferian \& Afessa 2006). Thus, there is a clear need for further systematic exploration of 225 human factors barriers to the implementation of new alert systems in the ICU setting, such as 226 the impact of workflow changes and the influence of method of alert delivery.

228 Implementation of automated detection and alert systems without consideration of these factors 229 is known to have the potential to result in alert fatigue (Singh et al. 2013), interruption (Hodgetts 230 \& Jones 2007), human error (Bates et al. 1998), and information overload (Stokstad 2001). 231 Recognition of the importance of alert fatigue in the hospital setting has increased significantly 232 in recent years (Herasevich et al. 2013). However, implementation of automated alert systems 233 generally must be performed in the context of information overload and complex task 234 interruption (Eppler \& Mengis 2004). It is also known that information overload can alter alert 235 perception in the medical setting (Glassman et al. 2006). This can cause clinicians to perceive 236 alert systems negatively and deter future use (Harrison et al. 2016). Thus, the task of generating 237 clinically meaningful alerts while concurrently minimizing information overload and task 238 interruption is challenging.

239

240 Clinician-participant comments provided valuable insight regarding preferences for method of 241 alert delivery. Although there was a clear preference for receiving urgent alerts through text 242 paging, additional investigation is required to specifically explore the rationale for this 243 preference. Understanding the rationale for this preference may reduce the barriers to 244 answering the primary objective of this study, which was comparison of time to severe sepsis 245 alert acknowledgement methods by critical care clinicians in the ICU setting. These secondary 
246 outcomes revealed important barriers to the inability to answer the primary outcome, which are 247 applicable and generalizable to future studies.

248 Even the reluctance of clinicians to participate in this research study, as revealed during the 249 post-study discussion, has the potential to confound interpretation of the results of this study. 250 Whether this reluctance is the result of information overload, the Hawthorne effect, and/or some 251 other factor(s), clinicians are currently struggling to find a balance between understanding of the 252 importance of their participation in clinical research studies and "study fatigue".

253

254 This study has several limitations. (1) This was a single-center study at an academic medical 255 center. Well-established biases and potential confounders are known to be present with this 256 particular study design (Straus et al. 2005). (2) Unlike the severe sepsis system alerts through 257 AWARE (yellow or green icon alerts), the severe sepsis alerts through text paging were 258 simulated. Comparing non-simulated alerts to simulated alerts may introduce additional 259 confounders into the interpretation of the results of this study. (3) Although not investigated in this study, the feasibility of severe sepsis alert delivery using an EHR-based, automated mobile app for smartphones has been validated (Dziadzko et al. 2016). (4) The significant range of clinical experience of clinician-participants introduces study bias. The potential application of this technology for the future of clinical practice and clinical research should not be ignored. Ultimately, a multi-center, non-simulated study in the ICU setting is required to address various aspects of these limitations.

\section{CONCLUSION}

268 It could not be determined whether an automated alert for severe sepsis reduced time to alert acknowledgement by critical care clinicians in the ICU setting compared to text paging. This was due to an extremely limited alert acknowledgement response rate using the severe sepsis alert

271 system compared to traditional text paging. Implementation barriers, including human factors- 
272 such as alert fatigue, interruption, human error, and information overload-were determined to 273 be an important source of this finding.

274

275 REFERENCES

276 Afessa B, Keegan MT, Hubmayr RD, Naessens JM, Gajic O, Long KH, and Peters SG. 2005.

277

278

279

280

281

282

283

284

285

286

287

288

289

290

291

292

293

294

295

296

297

298

299

300

301

302

303 Evaluating the performance of an institution using an intensive care unit benchmark. Mayo Clinic proceedings Mayo Clinic 80:174-180. 10.4065/80.2.174

Ahmed A, Chandra S, Herasevich V, Gajic O, and Pickering BW. 2011. The effect of two different electronic health record user interfaces on intensive care provider task load, errors of cognition, and performance. Critical care medicine 39:1626-1634. 10.1097/CCM.0b013e31821858a0

Bates DW, Leape LL, Cullen DJ, Laird N, Petersen LA, Teich JM, Burdick E, Hickey M, Kleefield S, Shea B, Vander Vliet M, and Seger DL. 1998. Effect of computerized physician order entry and a team intervention on prevention of serious medication errors. JAMA : the journal of the American Medical Association 280:1311-1316.

Bourgault AM, Heath J, Hooper V, Sole ML, Waller JL, and Nesmith EG. 2014. Factors influencing critical care nurses' adoption of the AACN practice alert on verification of feeding tube placement. Am J Crit Care 23:134-144. 10.4037/ajcc2014558

Dellinger RP, Levy MM, Rhodes A, Annane D, Gerlach H, Opal SM, Sevransky JE, Sprung CL, Douglas IS, Jaeschke R, Osborn TM, Nunnally ME, Townsend SR, Reinhart K, Kleinpell RM, Angus DC, Deutschman CS, Machado FR, Rubenfeld GD, Webb SA, Beale RJ, Vincent JL, and Moreno R. 2013. Surviving sepsis campaign: international guidelines for management of severe sepsis and septic shock: 2012. Critical care medicine 41:580637. 10.1097/CCM.0b013e31827e83af

Dziadzko MA, Harrison AM, Tiong IC, Pickering BW, Moreno Franco P, and Herasevich V. 2016. Testing modes of computerized sepsis alert notification delivery systems. BMC medical informatics and decision making 16:156. 10.1186/s12911-016-0396-y

ECRI-Institute. 2013. 2014 Top 10 List of Health Technology Hazards. Health Devices Journal 42.

Embi PJ, Jain A, and Harris CM. 2008. Physicians' perceptions of an electronic health recordbased clinical trial alert approach to subject recruitment: a survey. BMC medical informatics and decision making 8:13. 10.1186/1472-6947-8-13 
304 Eppler MJ, and Mengis J. 2004. The concept of information overload: A review of literature from

305

306

307

308

309

310

311

312

313

314

315

316

317

318

319

320

321

322

323

324

325

326

327

328

329

330

331

332

333

334

335

336

337 organization science, accounting, marketing, MIS, and related disciplines. Information Society 20:325-344. 10.1080/01972240490507974

Gill PS, Kamath A, and Gill TS. 2012. Distraction: an assessment of smartphone usage in health care work settings. Risk management and healthcare policy 5:105-114. 10.2147/RMHP.S34813

Glassman PA, Belperio P, Simon B, Lanto A, and Lee M. 2006. Exposure to automated drug alerts over time: effects on clinicians' knowledge and perceptions. Medical care 44:250256. 10.1097/01.mlr.0000199849.08389.91

Harrison AM, Gajic O, Pickering BW, and Herasevich V. 2016. Development and Implementation of Sepsis Alert Systems. Clinics in chest medicine 37:219-229. 10.1016/j.ccm.2016.01.004

Harrison AM, Herasevich V, and Gajic O. 2015a. Automated Sepsis Detection, Alert, and Clinical Decision Support: Act on It or Silence the Alarm? Critical care medicine 43:17761777. 10.1097/CCM.0000000000001099

Harrison AM, Thongprayoon C, Kashyap R, Chute CG, Gajic O, Pickering BW, and Herasevich V. 2015b. Developing the surveillance algorithm for detection of failure to recognize and treat severe sepsis. Mayo Clinic Proceedings 90:166-175. 10.1016/j.mayocp.2014.11.014

Herasevich V, Kor DJ, Subramanian A, and Pickering BW. 2013. Connecting the dots: rulebased decision support systems in the modern EMR era. Journal of clinical monitoring and computing. 10.1007/s10877-013-9445-6

Herasevich V, Pickering BW, Dong Y, Peters SG, and Gajic O. 2010. Informatics infrastructure for syndrome surveillance, decision support, reporting, and modeling of critical illness. Mayo Clin Proc 85:247-254.

HIMSS Analytics. 2016. The Hidden Cost of Pagers in Healthcare: How Outmoded Technology Is Draining Healthcare IT Budgets. TigerText.

Hodgetts HM, and Jones DM. 2007. Reminders, alerts and pop-ups: The cost of computerinitiated interruptions. Beijing. p 818-826.

Hooper MH, Weavind L, Wheeler AP, Martin JB, Gowda SS, Semler MW, Hayes RM, Albert DW, Deane NB, Nian H, Mathe JL, Nadas A, Sztipanovits J, Miller A, Bernard GR, and Rice TW. 2012. Randomized trial of automated, electronic monitoring to facilitate early detection of sepsis in the intensive care unit*. Critical care medicine 40:2096-2101. 10.1097/CCM.0b013e318250a887 
338 Kaukonen KM, Bailey M, Suzuki S, Pilcher D, and Bellomo R. 2014. Mortality related to severe

339

340

341

342

343

344

345

346

347

348

349

350

351

352

353

354

355

356

357

358

359

360

361

362

363

364

365

366

367

368

369

370

371 sepsis and septic shock among critically ill patients in Australia and New Zealand, 20002012. JAMA : the journal of the American Medical Association 311:1308-1316. 10.1001/jama.2014.2637

LaRosa JA, Ahmad N, Feinberg M, Shah M, Dibrienza R, and Studer S. 2012. The use of an early alert system to improve compliance with sepsis bundles and to assess impact on mortality. Crit Care Res Pract 2012:980369. 10.1155/2012/980369

Loo TS, Davis RB, Lipsitz LA, Irish J, Bates CK, Agarwal K, Markson L, and Hamel MB. 2011. Electronic medical record reminders and panel management to improve primary care of elderly patients. Archives of internal medicine 171:1552-1558. 10.1001/archinternmed.2011.394

Nelson JL, Smith BL, Jared JD, and Younger JG. 2011. Prospective trial of real-time electronic surveillance to expedite early care of severe sepsis. Annals of emergency medicine 57:500-504. 10.1016/j.annemergmed.2010.12.008

Pickering BW, Dong Y, Ahmed A, Giri J, Kilickaya O, Gupta A, Gajic O, and Herasevich V. 2015. The implementation of clinician designed, human-centered electronic medical record viewer in the intensive care unit: a pilot step-wedge cluster randomized trial. International journal of medical informatics 84:299-307. 10.1016/j.ijmedinf.2015.01.017

Pickering BW, Herasevich V, Ahmed A, and Gajic O. 2010. Novel Representation of Clinical Information in the ICU - Developing User Interfaces which Reduce Information Overload. Applied Clinical Informatics 1(2):116-131.

Sawyer AM, Deal EN, Labelle AJ, Witt C, Thiel SW, Heard K, Reichley RM, Micek ST, and Kollef MH. 2011. Implementation of a real-time computerized sepsis alert in nonintensive care unit patients. Critical care medicine 39:469-473. 10.1097/CCM.0b013e318205df85

Seferian EG, and Afessa B. 2006. Demographic and clinical variation of adult intensive care unit utilization from a geographically defined population. Critical care medicine 34:21132119. 10.1097/01.CCM.0000227652.08185.A4

Semler MW, Weavind L, Hooper MH, Rice TW, Gowda SS, Nadas A, Song Y, Martin JB, Bernard GR, and Wheeler AP. 2015. An Electronic Tool for the Evaluation and Treatment of Sepsis in the ICU: A Randomized Controlled Trial. Critical care medicine. 10.1097/CCM.0000000000001020

Singh H, Spitzmueller C, Petersen NJ, Sawhney MK, and Sittig DF. 2013. Information Overload and Missed Test Results in Electronic Health Record-Based Settings. JAMA internal medicine:1-3. 10.1001/2013.jamainternmed.61 
372 Steinman MA, Handler SM, Gurwitz JH, Schiff GD, and Covinsky KE. 2011. Beyond the

373

374

375

376

377

378

379

380

381

382

383

384

385

386

387

388

389

390

391

392

393

394

395

396

397

398

399

400

prescription: medication monitoring and adverse drug events in older adults. Journal of the American Geriatrics Society 59:1513-1520. 10.1111/j.1532-5415.2011.03500.x

Stokstad E. 2001. Information overload hampers biology reforms. Science 293:1609. 10.1126/science.293.5535.1609

Straus SE, Richardson WS, Glasziou P, and Haynes RB. 2005. Evidence-based medicine: how to practice and teach EBM.

Tchalla AE, Lachal F, Cardinaud N, Saulnier I, Bhalla D, Roquejoffre A, Rialle V, Preux PM, and Dantoine T. 2012. Efficacy of simple home-based technologies combined with a monitoring assistive center in decreasing falls in a frail elderly population (results of the Esoppe study). Archives of gerontology and geriatrics 55:683-689. 10.1016/j.archger.2012.05.011

Torio CM AR. 2013. National Inpatient Hospital Costs: The Most Expensive Conditions by Payer, 2011. HCUP Statistical Brief\#160.

Wagner MM, Eisenstadt SA, Hogan WR, and Pankaskie MC. 1998. Preferences of interns and residents for E-mail, paging, or traditional methods for the delivery of different types of clinical information. Proceedings / AMIA Annual Symposium AMIA Symposium:140-144.

Zhang J, Johnson TR, Patel VL, Paige DL, and Kubose T. 2003. Using usability heuristics to evaluate patient safety of medical devices. Journal of biomedical informatics 36:23-30.

\section{TABLE LEGEND}

Table 1: Number of shifts per clinician participant and number of participants per shift

Table 2: Comparison of alert response rate and median time to alert acknowledgement between the severe sepsis alert system through AWARE and simulated severe sepsis alerts through traditional text paging

\section{FIGURE LEGEND}

Figure 1: Schematic illustration of study design

Figure 2: Detailed daily email reminder to clinician participants with complete instructions 
401 Figure 3: Facsimile of the structured, mixed quantitative/qualitative survey provided to the 402 clinician participants with all quantitative results overlaid: median (IQR)

403 Figure 4: All qualitative responses to the structured, mixed quantitative/qualitative survey 404 reproduced in their entirety, including typographical errors 


\section{Table 1 (on next page)}

Table 1

Number of shifts per clinician participant and number of participants per shift 
1 Table 1: Number of shifts per clinician participant and number of participants per shift

\begin{tabular}{lccccccc}
\hline & $\begin{array}{c}\text { Total } \\
\text { AM } \\
\text { Shifts }\end{array}$ & $\begin{array}{c}\text { Total } \\
\text { PM } \\
\text { Shifts }\end{array}$ & $\begin{array}{c}\text { Total } \\
\text { Shifts }\end{array}$ & Shift, part 1 & $\begin{array}{c}\text { Number of } \\
\text { Providers }\end{array}$ & Shift, part 2 & $\begin{array}{c}\text { Number of } \\
\text { Providers }\end{array}$ \\
\hline NP/PA \#01 & 5 & -- & 5 & 02/05 Thu PM & 1 & $02 / 12$ Thu PM & 1 \\
NP/PA \#02 & 4 & -- & 4 & 02/06 Fri AM & 2 & $02 / 13$ Fri AM & 4 \\
NP/PA \#03 & 3 & 1 & 4 & 02/06 Fri PM & 2 & $02 / 13$ Fri PM & -- \\
NP/PA \#04 & 3 & -- & 3 & 02/07 Sat AM & 1 & $02 / 14$ Sat AM & 6 \\
NP/PA \#05 & 1 & -- & 1 & 02/07 Sat PM & 2 & $02 / 14$ Sat PM & -- \\
Attending \#01 & 7 & -- & 7 & 02/02 Mon AM & 4 & $02 / 09$ Mon AM & 2 \\
Attending \#02 & 1 & -- & 1 & 02/02 Mon PM & -- & $02 / 09$ Mon PM & 1 \\
Attending \#03 & -- & 1 & 1 & 02/03 Tue AM & 4 & $02 / 10$ Tue AM & 5 \\
Fellow \#01 & 7 & 2 & 9 & 02/03 Tue PM & 1 & $02 / 10$ Tue PM & -- \\
Fellow \#02 & 6 & -- & 6 & 02/04 Wed AM & 3 & $02 / 11$ Wed AM & 5 \\
Resident \#01 & 5 & 5 & 10 & 02/04 Wed PM & 1 & $02 / 11$ Wed PM & -- \\
Resident \#02 & 7 & 2 & 9 & 02/05 Thu AM & 2 & $02 / 12$ Thu AM & 4 \\
& & & & 02/08 Sun AM & 3 & $02 / 15$ Sun AM & 4 \\
& & & & 02/08 Sun PM & 1 & $02 / 25$ Sun PM & 1 \\
\hline
\end{tabular}




\section{Table 2 (on next page)}

Table 2

Comparison of alert response rate and median time to alert acknowledgement between the severe sepsis alert system through AWARE and simulated severe sepsis alerts through traditional text paging 
1 Table 2: Comparison of alert response rate and median time to alert acknowledgement between

2 the severe sepsis alert system through AWARE and simulated severe sepsis alerts through

3 traditional text paging

\begin{tabular}{llll}
\hline & Text paging (N=156) & AWARE (N=148) & p-value \\
\hline Alert response rate (N) & $51 \%(80)$ & $3 \%(5)$ & 0.001 \\
\hline $\begin{array}{l}\text { Median time to alert } \\
\text { acknowledgement (IQR) }\end{array}$ & 2 mins (1 to 32) & 274 mins (130 to 517) & 0.053 \\
\hline
\end{tabular}

4 
Figure 1

Figure 1

Schematic illustration of study design

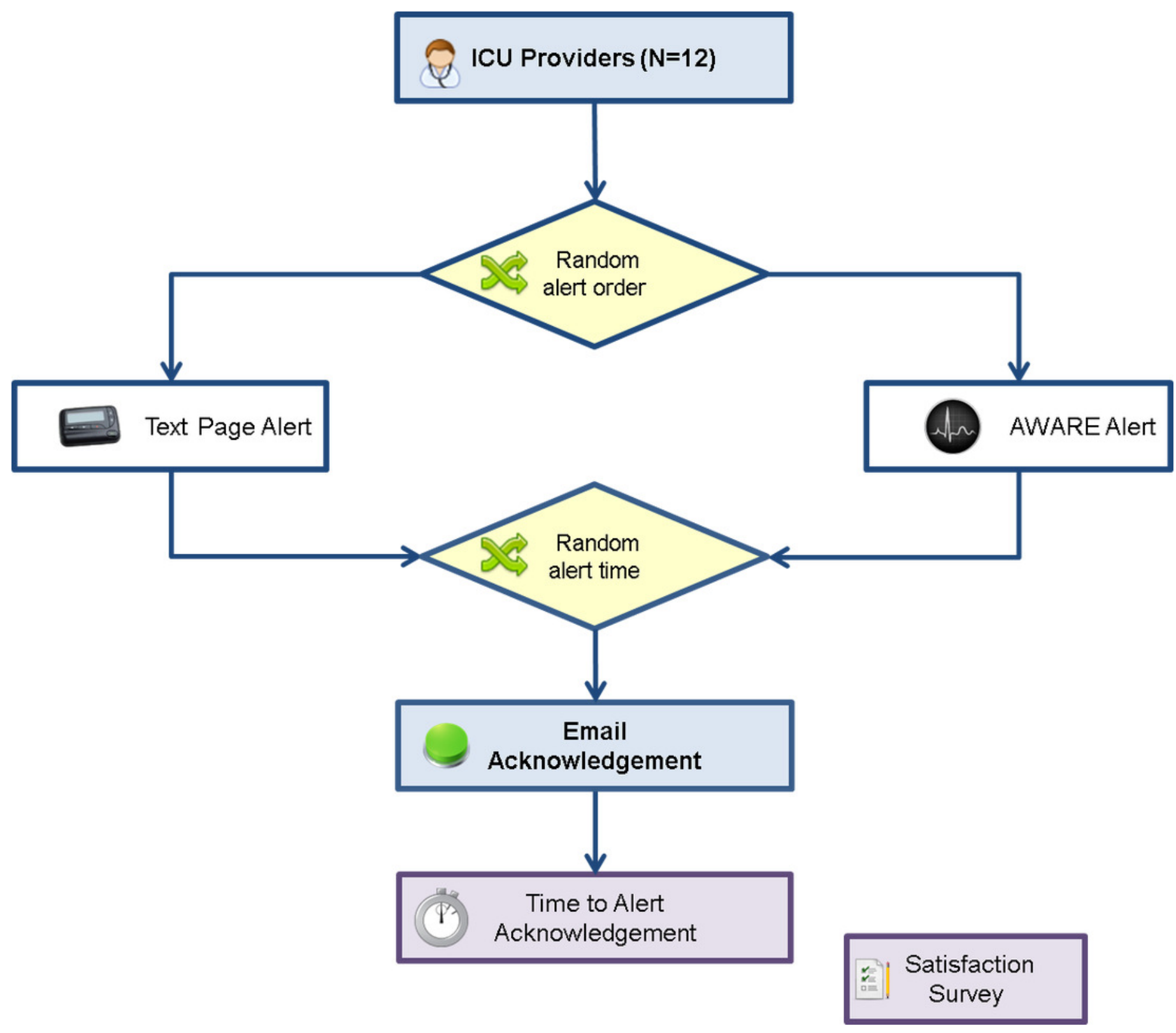




\section{Figure 2 (on next page)}

Figure 2

Detailed daily email reminder to clinician participants with complete instructions. 


\section{Greetings,}

Thank you for agreeing to participate in our study (IRB 13-003325) to test mechanisms of alert delivery (text page vs EMR-Aware).

- You will receive 1-3 random text pages per shift in MB 6B/G for the next 4 weeks. Upon receipt, please directly email me with these Alert Numbers. Feel free to use this email as a reference point for responding, but please do not Reply All.

- You are al so asked to email me the patient ID and icon color every time you identify a new* Yellow or Green sepsis alert icon in AWARE for any patient in MB 6B/G during your shifts, even if this patient is not assigned to your care team. *Please include icons al ready active at the start of your shift.

- One research restriction: we ask that you do not email via smartphone. Desktop, laptop/CoW, and iPad are all acceptable.

I will not respond to your acknowledgement emails, but please feel free to contact me directly if there are any questions or concerns at any point throughout the duration of this study. At the conclusion of this study, we will coordinate a pizza party through the Division of Critical Care to celebrate. 


\section{Figure 3 (on next page)}

Figure 3

Facsimile of the structured, mixed quantitative/qualitative survey provided to the clinician participants with all quantitative results overlaid: median (IQR) 
Please provide at least one suggestion for improving alert/notification delivery:

Attending 01: Having a nurse pay closer attention to the patient and be less involved in online shopping/facebook checking (something I see way too often) and not to hesitate to notify the clinician when something is not right. Sometimes when I pass by, there is no one around and patient is hypotensive and looks very ill, and no one is aware of it because nurse is on a break and the other nurse doesn't know the patient and is otherwise occupied.

Attending 02: Text page for urgent issues should work. For non-urgent issues, creating more "in-baskets" or messages will create fatigue

Attending 03: Text message to phone or pager (phone preferred). Alternative would be a notification to phone through Synthesis or whatever the future app EMR might hold. This would be the most preferred for those of us that don't live glued to scanning the AWARE home screen. Fellow 01: When notifying with pager add patient details too like room number, clinic number etc Fellow 02: Most of residents do not use AWARE regularly. AWARE compliance need to be improved if messages are to be delivered by AWARE

Resident 02: I prefer pages. I'm still not used to using AWARE. If there is a truly urgent matter, I prefer a phone call to the portable phone.

NP/PA 01: I like paging as I always have it with me. I don't always have AWARE even with my iPAD that I have to log in to every 5-10 minutes.

NP/PA 02: No E-mial

NP/PA 03: Please use the pager for all urgent clinical alerts. Providers do not always have their Ipads or access to a computer, particularly during when spending time in patient rooms and in discussion with families.

NP/PA 04: Paging is best as I do not always have up e-mail if with patients, etc.. Text pages worked well. Just for thought: what if page did not go through... should there be a second option in place? ${ }^{*}$ Duplicate Page ${ }^{* *}$ notification

NP/PA 05: It is more difficult to keep up in the notifications through AWARE, especially if you don't always use the aware program throughout the day. I feel that the paging system would make me more prone to look at aware for a sepsis alert, but I feel that it would become a bit much and put to the side if I received a page for every sepsis alert everyday shift, all day. A suggestion would be to page with red alerts only or if no one has "claimed" a sepsis warning patient to remind us.

Please provide any additional comments you wish:

Attending 03: Native AWARE app would be great, with swiping between screens to move from organ system to organ system for those of us that are not in love with big clunky iPads ... yes, even the ipad mini is too big for my taste.

NP/PA 01: Paging will get me alerted to soonest.

NP/PA 04: None, I hope my participation helped with the survey. I became more "aware" of the critically ill patients by participating - not only on my team but my colleagues' team as well. Became more mindful to offer assistance.

NP/PA 05: Due to my schedule during the trial period, I only worked a couple of shifts, which may have altered my input as I did not get accustom to it or have a lot of time working with the paging system. Thanks. 


\section{Figure 4 (on next page)}

Figure 4

All qualitative responses to the structured, mixed quantitative/qualitative survey reproduced in their entirety, including typographical errors 
Age (years): 41.0 (29.3 to 43.5)

Years in practice: 6.5 (2.3 to 14.8)

Years, months, weeks, or days working with the Mayo Clinic EMR: 2.5 years (1.1 to 13.3)

Years, months, weeks, or days working in the medical ICU: 1.1 years (0.7 to 2.8)

Years, months, weeks, or days working with AWARE: 1.4 years (0.6 to 2.5)

Please rate 1 through 5: 1 (Never), 2 (Rarely), 3 (Sometimes), 4 (Frequently), or 5 (Always)

- Was notification by paging disruptive? 3 (2 to 3 )

- Was notification by AWARE disruptive? 2 (1 to 2 )

- Was paging acknowledgment difficult? 3 (1 to 4)

- Was AWARE acknowledgment difficult? 3 (1 to 4)

Please rate 1 through 5: 1 (Always paging), 2 (Mostly paging), 3 (No preference), 4 (Mostly AWARE), 5 (Always AWARE)

- Which would be your preferred method of non-urgent alert/notification? 2 (1 to 5)

- Which would be your preferred method of urgent alert/notification? 1 (1 to 2)

For the questions below, if multiple options are preferred, please select more than one.

- The best method for non-urgent clinical alert/notification is (circle or bold)

Paging (6) AWARE (5) Email (3) Phone call (0) Text message (2) Other: none (1)

- The best method for urgent clinical alert/notification is (circle or bold)

Paging (11) AWARE (1) Email (0) Phone call (2) Text message (1) Other: (0)

Please provide at least one suggestion for improving alert/notification delivery:

\section{1 out of 12 participant provided a response.}

Please provide any additional comments you wish:

4 out of the 11 participants above also provided an additional comment. 